\title{
Trazos para un mapa de la investigación sobre juventud en América Latina
}

\section{José Antonio Pérez Islas}

Instituto Mexicano de la Juventud

Centro de Investigación y Estudios sobre Juventud

japerez@imjuventud.gob.mx; perezislas@yahoo.com

\section{Resumen}

El desarrollo de la investigación sobre juventud en América Latina en los últimos veinte años ha generado una amplia producción en distintos rubros. En este artículo se hace un recuento y análisis de los principales esfuerzos en la materia y sus discusiones centrales a partir de los informes regionales, los estados del arte nacionales, las encuestas nacionales de juventud, las publicaciones periódicas especializadas en la materia y los principales grupos de trabajo. Sin embargo, la fragmentación, la escasa institucionalización, la poca vinculación entre los agentes sociales involucrados (investigadores e instituciones académicas, gobiernos y organizaciones civiles) entre otros, han impedido la consolidación de un campo propio (como lo plantea Bourdieu), que, en el contexto de una región como la latinoamericana (la importancia cuantitativa de las jóvenes y los jóvenes y la presencia de retos inéditos que se acentúan en las nuevas generaciones), son de vital relevancia para pensar desde miradas renovadas a los sectores juveniles. Hacer un alto en el camino y otear el horizonte y las condiciones prospectivas de la producción en juventud es el objetivo de este trabajo.

Palabras clave: juventud, investigación y estado del arte, América Latina.

\section{Abstract. A bluepoint for a map of youth research in Latin America}

In the last 20 years, youth research development in Latin America has generated a broad production in different fields. This article takes account and analyses the most important efforts on this matter and their central discussions, having as sources regional reports, national states of art, national youth surveys and periodical specialized journals, as well as the main workgroups in the matter. Nevertheless, the fragmentation, incipient institutionalization and the scarce links between the social agents involved (researchers, academic institutions, governments and civil society organizations, among others) has hindered the consolidation of a field of study for itself — as Bourdieu proposes - which, in the context of a region such as Latin America (with the quantitative importance of youth and the existence of rising challenges accentuated in new generations), are of vital relevance to think youth sectors from renewed approaches. To make a stop at the road and take a look at the horizon and the prospective conditions of youth research production, are the objectives of this paper.

Key words: youth, investigation and stat of art, Latin America. 


\section{Sumario}

I. Los informes latinoamericanos y los Estados del Arte

II. Otros lugares de producción de conocimiento
III. Un balance provisional

Bibliografía

«¿Qué sabemos de los jóvenes?», fue la pregunta central en un seminario latinoamericano realizado en Bogotá, Colombia, en 1996, que, independientemente de la calidad de los trabajos presentados (en general con muy buena factura) y del libro colectivo que se produjo —uno de los más conocidos y citados en el continente durante los años siguientes - ${ }^{1}$, es una muestra de lo que podría ser la historia reciente de la investigación sobre juventud en América Latina (AL): estudiosos de primera línea, académicamente muy sólidos, desarrollando trabajos novedosos de investigación, pero con una falla de origen: una gran fragmentación y pocas posibilidades de vinculación, discusión y construcción colectiva; este seminario pretendía un estado del arte de lo investigado y únicamente se alcanzó una aproximación de los avances que cada participante había obtenido desde su experiencia individual. En el extremo está otro libro recién publicado ${ }^{2}$ (que es la mejor forma de objetivación del avance de lo producido), donde se muestra una comunidad académica más articulada, que cuando menos lleva quince años debatiendo y compartiendo categorías y resultados de sus indagaciones; dicha comunidad está integrada por investigadores de México y Cataluña, es decir, dos regiones atravesadas por un océano.

¿Por qué es menos difícil articular grupos de investigadores, a primera vista con realidades más distantes, que a otros, que habitan un mismo continente y que se encuentran con situaciones relativamente compartidas? ¿Qué ha pasado en AL entre el boom del Año Internacional de la Juventud (AIJ) y los primeros años del nuevo siglo? ¿Han existido avances? Intentaremos dibujar algunas coordenadas que permitan ubicar muy esquemáticamente la trayectoria de la investigación sobre los jóvenes en el continente, y lanzar algunas hipótesis al respecto a manera de balance.

Para lograr nuestro objetivo, que siempre será ilustrativo y no exhaustivo, pasaremos revista, en primer lugar, a los diversos esfuerzos que se han desarrollado para evaluar el avance de la investigación sobre la población juvenil; a continuación, se analizarán algunos espacios que pueden ser otros indicadores de lo logrado (revistas y programas especializados, encuestas nacionales específicas, grupos de trabajo y espacios institucionales). Terminaremos con

1. Humberto, J.; Cubides, C. y otros (editores), Viviendo a toda. Jóvenes, territorios culturales y nuevas sensibilidades, Santafé de Bogotá, Siglo del Hombre Editores-Fundación Universidad Central, 1998.

2. Rossana Reguillo y otros (coords.), Tiempo de Hibridos. Entresiglos, Jóvenes México-Cataluña, México, SEP-IMJ-SGJ-CIIMU, 2003. 
un balance de la narrativa realizada y de ciertos desafíos por encarar en los próximos años.

Habría que empezar diciendo en descargo, que AL es un constructo, que si bien tiene un origen cuasi común, comparte una masa territorial y los países tienen ciertas semejanzas en sus condiciones geopolíticas; sin embargo, es difícil hablar de un conjunto compacto, dado que existen varias Américas Latinas, que pueden ser clasificadas según lo que queramos medir. Por ejemplo, existen países con semejantes niveles de pobreza que no necesariamente tienen el mismo nivel de desarrollo económico, o que comparten similar nivel de crecimiento económico, pero no de institucionalización en su democracia. Un mismo acontecimiento, como la crisis económica de la década de los ochenta, tuvo diferente impacto y distinta estrategia para enfrentarse a ella según el país de que se tratase. Esta complejidad varía de acuerdo con la subregión o el área subnacional que se refiera y que deriva de las circunstancias histórico-culturales específicas y de los patrones de cambio social que se estén desarrollando, que, aunada a la creciente internacionalización de los procesos económicos y sociales, produce la polarización, fragmentación e intensificación de esas ancestrales heterogeneidades ${ }^{3}$. No obstante, de manera paradójica, AL y los latinoamericanos conservamos una identidad, que nos concede la posibilidad de hablar de ella (de nosotros) como una totalidad, sobre todo, frente al «otro».

Es en este contexto que se debe ubicar nuestra narración, pero, además, también habría que referirse a un siguiente círculo, donde la investigación (sobre todo la social) se ubica en uno de los últimos renglones presupuestales de las instituciones públicas y privadas, gubernamentales y no gubernamentales, internacionales y nacionales. Y la cuestión no termina ahí, como lo iremos comprobando a lo largo de este texto, la investigación social en juventud específicamente tampoco se ubica en el mainstream de las instituciones académicas o de los grupos hegemónicos de investigadores, por lo tanto, está fuera de la agenda prioritaria que define recursos humanos, económicos y materiales, sin embargo..., se mueve.

\section{Los informes latinoamericanos y los estados del arte}

\section{Los informes regionales}

La historia de la investigación sobre juventud en AL se ha documentado poco, el trabajo más relevante por su visión de conjunto se debe a Cecilia Braslavsky, quien, en 1987, realizó un análisis de lo acontecido hasta ese año ${ }^{4}$, en el marco

3. Amparo Méndez-Carrión y Fernando Bustamante, «Purposes and methods of Intraregional Comparison", en Meter H. SMITH (coord.), Latin America in Comparative Perspective. New approaches to methods and analysis. Westview Press, Inc., USA, 1995, p. 60.

4. Cecilia BRASLAVSKY, «Estudios e investigacionmes sobre juventud en América Latina: balance y perspectivas», en, Ernesto RodríGUeZ y Ernesto OTTONE (comp.), Mitos, certezas y esperanzas. Tendencias de las investigaciones sobre juventud en América Latina, Montevideo, CELAJU-UNESCO, 1989, p. 17-48. 
de un proyecto que buscó articular la primera Red Latinoamericana de Expertos sobre el tema; y donde la mayor parte de los trabajos publicados giran en torno al avance logrado en diversos países del continente. En este artículo, Braslavzky planteaba que habían existido tres primeras grandes etapas:

\section{La ensayística (1930-1960)}

Donde destacaron dos grupos: los precursores de la teoría generacional, de origen positivista, que siguiendo a Ortega y Gasset, asumieron que, en la dinámica social, las generaciones actuarían como el agente principal. El ejemplo más claro se encuentra en el español Julián Marías, que, desde Argentina, desarrollaría este carácter generacional como forma de vida con una determinación histórica y no biológica. El segundo grupo, que Braslavzky llama histórico-crítico, está representado por José Martí, Aníbal Ponce y Vicente Lombardo Toledano, que, en conferencias y cursos, ponen en evidencia el conocimiento y la preocupación que tenían sobre los jóvenes de sus países.

\section{El predominio de la sociología (1960-1980)}

En este periodo se inician trabajos propiamente de investigación, también se pueden distinguir dos grupos: el primero que se ocupa sobre todo de los jóvenes universitarios y el segundo de los jóvenes de un modo más general, ambos de alguna manera están vinculados al Instituto Latinoamericano de Planificación Económica y Social (ILPES). Desde aquí surge el primer estado del arte sobre juventud, realizado por José Medina Echavarría en 1967, quien concluye: "gocemos al menos la claridad de la docta ignorancia: saber que nada sabemos con cierta precisión no ya por la generación más joven, sino tampoco por las intermedias que nos son más próximas». Destacan, en estos dos grupos, Aldo Solari y sus abordajes de los jóvenes como «categoría estratificacional» (primer nivel) y los jóvenes como ciudadanos (segundo nivel); seguido por uno de los textos ya clásicos de Gurrieri, Torres-Rivas y otros autores: Estudios sobre la Juventud Marginal Latinoamericana ${ }^{5}$, quienes analizan a estos sectores en los países de Chile, Perú y El Salvador.

\section{El periodo del Año Internacional de la Juventud (1982-1986)}

Braslavzky llama a esta etapa, la "contemporánea», donde la producción se multiplica varias veces comparativamente a lo que se había generado en las dos anteriores. Según la autora, el origen de este inusitado interés se debe a cuatro factores: las transformaciones de la juventud, el festejo del AIJ, las crisis de las sociedades latinoamericanas y el renacimiento de las democracias en el continente. Un apoyo importante para lograr esto fueron los apoyos recibidos para la serie de estudios realizados por parte de varias institu-

5. A. GURRIERI y otros, Estudios sobre la Juventud Marginal Latinoamericana, Siglo XXI, México, 1971. 
ciones del sistema de las Naciones Unidas, pero sobre todo de la Comisión Económica para América Latina y el Caribe (CEPAL). Un buen número de los estudios de esta etapa se basa sobre todo en fuentes secundarias (censos, encuesta de hogares, estadísticas educativas, etc.) y en muchos casos son investigaciones sobre «menores» y no propiamente sobre jóvenes. Definitivamente, durante este lapso se instauran los estudios sectoriales: el empleo y los jóvenes, la salud juvenil, la participación política de los jóvenes, etc. Un segundo grupo aunque más escaso es el que utiliza trabajo de campo, fundamentalmente entrevistas y encuestas en cuatro vertientes: jóvenes de poblaciones urbano-marginales, estudiantes de nivel medio, universitarios y proyectos de trabajo con jóvenes.

Un segundo documento que de alguna manera hace otra revisión sobre el estado de la investigación en el continente, es el elaborado por Ernesto Rodríguez y Bernardo Dabezies, por encargo de la III Conferencia Iberoamericana de Juventud celebrada en 1989, se titula, Primer Informe sobre la Juventud de América Latina ${ }^{6}$ y, según lo manifiestan los autores en el prólogo de dicha publicación, «se convertirá en la columna vertebral sobre la cual la conferencia articulará el proceso investigativo en los años sucesivos».

El documento aborda fundamentalmente los resultados logrados y los principales hallazgos en la población juvenil, más que el tipo y la caracterización de las investigaciones que se realizaron, su metodología o sus presupuestos conceptuales. Como sus autores lo señalan, es la continuación del libro Mitos, certezas y esperanzas, y su logro central es la conjunción y sistematización de todo el material que existía en el momento y que se hallaba disperso. El documento se estructura en cinco partes: la primera que aborda el contexto de modernización (1950-1980) y crisis (1980-1990) de América Latina, el desarrollo del concepto de juventud y una caracterización demográfica de la población joven; la segunda, se refiere a las tres grandes áreas sectoriales vinculadas con los jóvenes: educación, empleo y salud; la tercera parte, se detiene en cuatro sectores juveniles con mayor claridad delimitativa en los estudios: universitarios, juventud popular urbana, rural y mujeres jóvenes; la cuarta parte, toca tres aspectos particulares de los comportamientos juveniles: la cultura y el deporte, la violencia y las formas participativas en las democracias reinstauradas, finaliza con una sección donde se plantean los desafíos de las futuras investigaciones en el continente.

Éste es un documento que logra sintetizar muchos de los avances de investigación en la región en su momento que concluyen en siete desafíos para la década de los noventa: desarrollar una estadística básica de primera mano; diversificar los análisis de sectores juveniles específicos; apoyar los procesos de indagación en los países con poca actividad; ampliar la cantidad de disciplinas involucradas en la investigación de juventud para generar trabajos inter-

6. Ernesto Rodríguez y Bernardo DABEZIES, Primer Informe sobre la Juventud de América Latina, 1990, Conferencia Iberoamericana de Juventud, Madrid, 1991. 
disciplinarios; establecer fuertes relaciones con el campo de las políticas de juventud; generar espacios más institucionales para que los equipos tengan un espacio más estable de desarrollo, y consolidar los avances teóricos en la materia.

Para lograr lo anterior, Rodríguez y Dabezies proponen el Programa de investigaciones básicas sobre juventud para los noventa, bajo dos ejes: el primero se refiere a un aspecto central producido en la ya famosa «década perdida de los ochenta" en AL, la exclusión, que si bien afecta a amplios sectores de la población, es en los jóvenes donde mejor se catalizan procesos que les limitan sus expectativas de vida y de participación; mientras que el segundo eje gira en torno a las dimensiones estructurales de la inserción social de los jóvenes con tres prioridades: la relación entre educación y empleo; las actitudes y los comportamientos juveniles y su relación con los agentes (instituciones) de socialización; así como la profundización sobre sectores específicos. Concluyen con algunas propuestas para la conjunción de metodologías y de técnicas cuantitativas y cualitativas; para la difusión de los resultados y para la consecución de fuentes de financiamiento.

Hasta aquí los esfuerzos por realizar balances regionales sobre el estado de la investigación sobre juventud. Otros tres textos importantes aparecerán posteriormente a nivel de AL (uno de ellos nunca vio la luz pública por problemas políticos), los cuales buscarán realizar un diagnóstico de la condición juvenil en el continente y cómo las políticas públicas pueden incidir en este sector.

El primero de ellos fue un gran esfuerzo coordinado por Ernesto Rodríguez, apoyado por la Organización Iberoamericana de Juventud (OIJ) y financiado por el Centro Internacional de Investigaciones para el Desarrollo (CIID) de Canadá, que, durante $1994-1995^{7}$, reunió a veintiséis investigadores de América Latina para realizar veinte informes nacionales, cuatro comparativos subregionales, tres estudios en profundidad y un informe final. Su enfoque definitivamente estaba centrado en analizar las políticas de juventud, pero a lo largo de los trabajos realizados se pueden apreciar los avances en el conocimiento de los jóvenes, desgraciadamente desavenencias entre la conducción política y la académica impidieron la publicación integral de los textos elaborados $^{8}$.

El segundo trabajo aparece en el año 2000 bajo los auspicios de la Organización de las Naciones Unidas ${ }^{9}$, donde se retoman muchos de los resultados no publicados en el proyecto antecedente OIJ-CIID, sin embargo, como buen documento de la CEPAL, se centra sobre todo en la información esta-

7. OIJ-CIID, Proyecto de investigación: Políticas de Juventud en América Latina: evaluación y reformulación, Montevideo, 1994 (inédito).

8. Sólo algunos fueron publicándose a iniciativa de sus autores, por ejemplo: Guillermo DÁvalos y Huáscar CAJÍAs, «Juventud boliviana: utopías y realidades», El Debate Boliviano, no 12, Seamos, La Paz, 1995, p. 53-92.

9. ONU-CEPAL, Juventud, población y desarrollo en América Latina y el Caribe. Problemas, oportunidades y desafios, CELADE, CEPAL, FNUAP, Santiago de Chile, 2000. 
dística y demográfica que brindan fuentes secundarias; su estructura se vincula a las tradicionales segmentaciones sectoriales (educación, salud, empleo), aunque ya aparecen nuevas temáticas como la migración, el rock y las nuevas formas de expresión y representación juvenil.

El tercer informe latinoamericano corresponde a 2004, también producido desde la CEPAL y coordinado por Martín Hopenhayn ${ }^{10}$. Este texto tiene varias novedades: la primera, que recopila información no sólo de Latinoamérica, sino también de España y Portugal, ambos miembros de la OIJ; la segunda, que abre un apartado donde se analizan las relaciones de los jóvenes con sus familias; de igual manera, incluye el tema de la pobreza y cómo afecta a la población juvenil según su sexo, región (rural o urbana y tipo de hogar); también incorpora un capítulo dedicado a los consumos culturales y a las sensibilidades juveniles, para terminar con una propuesta de indicadores que den seguimiento a la situación de los jóvenes iberoamericanos. A las fuentes tradicionales, le suman los resultados obtenidos por las encuestas nacionales de juventud realizadas en diversos países.

Obviamente, adicionalmente a los balances generales aquí expuestos han sido elaborados otros, pero siempre con temáticas muy concretas; quizá los más socorridos se refieren a las áreas de sexualidad y salud reproductiva ${ }^{11}$ y a las vinculadas con el trabajo y la ocupación ${ }^{12}$, muchos de ellos realizados gracias a los organismos nacionales o regionales especializados en ellos. Por desgracia, en otros ámbitos como la cultura, la participación, etc. no existen este tipo de instituciones ni de proyectos.

\section{Los estados del arte}

Otros esfuerzos por realizar este ejercicio autorreflexivo que significan los estados del arte se han producido a nivel nacional o local. Quizá el precursor sea el caso mexicano, que ya en 1985 realizó una primera revisión, cuyos resultados sólo se publicaron sintéticamente ${ }^{13}$. Once años más tarde, en 1996, se invitó a siete investigadores para que, con una misma metodología, hicieran una evaluación sobre lo que se conocía en ese momento de los jóvenes en igual

10. OIJ-CEPAL, La juventud en Iberoamérica. Tendencias y urgencias, Santiago de Chile, 2004.

11. Claudio STERN y Elizabeth GARCÍA, Acervo documental sobre sexualidad y salud reproductiva de adolescentes y jóvenes, Documentos de Trabajo no 2, Colegio de México-Programa Salud Reproductiva y Sociedad, México, 2000.

12. CINTERFOR-OIT, Empleo y capacitación de jóvenes en América Latina: situación, perspectivas y politicas alternativas, Montevideo, 1988.

13. J. A. PÉREZ Islas y Sergio ZERMEÑO, «La juventud mexicana: una discusión sobre su conocimiento», en Ernesto RodrígueZ y Ernesto OTTONe (comps.), op. cit., p. 119-145. En este texto se concentró el recuento de veinticinco años de investigación e historia de la juventud en México, de 1960 a 1985, sobre problemas específicos de interés para los investigadores en ese momento: empleo y educación, juventud fronteriza, comunicación y cultura en los jóvenes, salud, recreación y tiempo libre, mujer joven, bandas juveniles, juventud rural y participación política de los jóvenes. 
número de temáticas: educación y empleo juvenil; cultura juvenil y medios; valores y religión en los jóvenes; sexualidad juvenil, juventud y adicciones; formas de agregación juvenil, y, participación política y ciudadana de los jóvenes ${ }^{14}$. Dichos textos fueron comentados y discutidos en una reunión nacional que a su vez sirvió para establecer la red de investigadores en el país.

El balance permitió establecer algunas tendencias, donde destacan ${ }^{15}$ :

- A partir del estudio de las diferentes agregaciones juveniles, y después de un cierto estancamiento en las discusiones en torno al concepto «juventud», en México se está produciendo una nueva reflexión teórica a partir de la construcción de la categoría de «culturas juveniles», con dos vertientes complementarias: la primera que surge de la articulación de los elementos de generación, género, clase, etnicidad, territorios y estilos ${ }^{16}$, y la segunda, a partir de la conformación de «identidades o identificaciones juveniles» ${ }^{17}$ y de "la historia cultural de la juventud», que se concreta en la investigación etnográfica de esas identidades juveniles ${ }^{18}$. Estas perspectivas superan las aporías que se habían encontrado en torno a conceptualizaciones generales que opacaban las especificidades de los grupos juveniles.

- Por otra parte, desde el ámbito cultural más estrictamente hablando, las preocupaciones giraron en torno a las manifestaciones ligadas a la música, todavía hegemonizadas por el rock $^{19}$, pero abriéndose a otras corrientes musicales ${ }^{20}$. Las transformaciones tecnológicas y sus impactos en las nuevas generaciones (particularmente la informática e Internet) son otros temas que están empezando a cobrar importancia dentro de la comunidad mexicana de investigadores y que quizá se vuelvan centrales en los años por venir.

14. J. A. Pérez Islas y Elsa P. Maldonado Oropeza (coords.), Jóvenes: Una evaluación del conocimiento. La investigación sobre juventud en México 1986-1996 (2 tomos), Causa JovenCIEJ, México, 1996. Tres años más tarde se reeditaría con la actualización de algunos textos.

15. J. A. PÉREZ IsLAS, «Introducción», en J. A. PÉREZ IsLAS (coord.), Jóvenes: Una Evaluación del Conocimiento. La investigación sobre juventud en México 1986-1999 (2 tomos), IMJ-CIEJ, México, 2000 (edición corregida y aumentada), p. 9-10.

16. Carles FeiXA, El Reloj de Arena. Culturas juveniles en México. Causa Joven-CIEJ, México, 1998.

17. José Manuel VALENZUELA, "Culturas juveniles. Identidades transitorias», en JOVENes. Revista de Estudios sobre Juventud. Causa Joven-CIEJ, cuarta época, año $1, \mathrm{n}^{\circ}$ 3. México DF, enero-marzo, 1997, p. 12-35.

18. Rossana REGUILLO. "Culturas Juveniles. Producir la identidad: un mapa de interacciones», en JOVENes. Revista de Estudios sobre Juventud. Causa Joven-CIEJ, cuarta época, año 2, no 5, México DF, julio-diciembre, 1997, p. 12-31.

19. José Manuel VAlenZuela y Gloria GonZÁlez. Oye Como va. Recuento del rock tijuanense, Instituto Mexicano de la Juventud-CIEJ, México, 1999.

20. El número dedicado a «Música y culturas juveniles», de JOVENes. Revista de Estudios sobre Juventud. Causa Joven-CIEJ, cuarta época, año 2, no 6, México DF, enero-marzo, 1998. 
- Posiblemente el mejor sistema de investigación constituido en México sea en materia de adicciones, que sigue identificándose casi mecánicamente como una problemática juvenil, por lo que ha concentrado la preocupación de muchos sectores sociales, sobre todo por el incremento en el consumo de substancias «duras» en los últimos años. No obstante, que los métodos cualitativos comienzan a usarse en este rubro, todavía predominan los enfoques cuantitativos y epidemiológicos.

- Un tema verdaderamente emergente en la cuestión juvenil es el de la sexualidad, impulsado por la preocupación en torno al sida, pero que está logrando acercarse a otra serie de temas fundamentales. Para plantear nuevas formas de comprender las dinámicas de diferentes sectores de jóvenes; sólo quizá habría que empezar a incorporar con mayor fuerza los aspectos afectivos en las indagaciones futuras.

- En el lado opuesto, se encuentra la investigación sobre la relación entre educación y empleo, que, contradictoriamente, siendo un aspecto directamente vinculado con los jóvenes, es un tema sin visión juvenil; esto quiere decir que si bien existe una amplia producción en materia de investigación educativa, pocas veces se ve al estudiante como joven que interactúa en múltiples espacios sociales de manera paralela a la escuela. Pero lo más preocupante está en el ámbito laboral, que sigue siendo una verdadera asignatura pendiente, donde prácticamente no existen estudios que muestren la especificidad de los problemas que enfrentan a los jóvenes en los diversos mercados de trabajo.

- Otra vertiente que se está replanteando totalmente es la de la participación política de los jóvenes. Se superan las limitaciones que implicaba concebirla sólo vinculada a la esfera de la política formal tradicional (partidaria y electoral). Las nuevas perspectivas en torno a la ciudadanía juvenil están mostrando otros significantes de las prácticas y concepciones de los jóvenes, que mucho tienen que ver con la irrupción de lo que se ha dado por llamar «sociedad civil»; no obstante, falta mucha indagación empírica sobre el tema.

La segunda indagación nacional se hizo en Uruguay en $2003^{21}$, aunque entrelazando los avances de juventud con los de mujer; para el caso de los estudios de jóvenes se diferencian cuatro periodos: 1950-1967, cuando su problema central es la educación, por lo tanto aparecen como estudiantes; 19681973, aparece la emergencia social de los jóvenes y los estudiantes se convierten en actores políticos; entre este periodo y el siguiente se produce una ausencia total de estudios sobre los jóvenes; 1974-1984, es el lapso donde las preocupaciones sobre los jóvenes se vuelcan sobre el trabajo y la desocupación como expresión del estancamiento de la estructura social; finalmente, 1985-1989,

21. Beatriz LOVESIO y Nilia VISCARDI, «Los estudios de la mujer y de los jóvenes en la construcción del conocimiento sociológico uruguayo", Revista de Ciencias Sociales, año XVI, no 21, Universidad de la República-Facultad de Ciencias Sociales-Departamento de Sociología, Montevideo, agosto 2003, p. 79-109. 
es el periodo de los movimientos sociales y de la exclusión y la violencia y cuando se consolida una sociología de la juventud en Uruguay.

Las conclusiones a que llegan las autoras son muy interesantes, porque, en alguna medida, reproducen lo acontecido en diversos países del continente.

El primer momento de los estudios sobre juventud de Uruguay parte de una mera "delimitación etárea, fisiológica y demográfica», los primeros en ser conceptualizados son los estudiantes, como un grupo social en etapa de inserción y socialización, y de ahí se pasa a la categoría de "moratoria», en tanto «espacio temporal de transición que la sociedad brinda a un determinado sector social, espacio situado entre la infancia y la edad adulta» ${ }^{22}$.

Adicionalmente, los conflictos de clase y los procesos de diferenciación juvenil son un eje que se utiliza "para mostrar las diferencias entre jóvenes, y no para mostrar cómo en determinadas clases y grupos sociales las relaciones entre generaciones son diferentes, explicando [...] el proceso por el cual la juventud es un problema de las clases medias y sectores adyacentes» ${ }^{23}$.

El tercer estado del arte conocido se refiere a Chile ${ }^{24}$, otro país donde se ha desarrollado una buena masa crítica en materia de generación de conocimiento en torno a la condición juvenil; la historia es muy similar, se pueden detectar tres periodos: el primero, que va de la década de los años sesenta hasta el golpe militar (1973), donde destaca «la figura del joven estudiante como el principal protagonista del cambio» ${ }^{25}$.

La segunda época va de finales de los setenta hasta 1989, donde la reflexión se daba a partir de las organizaciones de la sociedad civil (OSC) y de la misma Iglesia católica, y se centra en un nuevo grupo «la juventud urbano poblacional». Oscar Dávila ${ }^{26}$, director de la revista Última Década (sobre la cual más adelante hablaremos), nos narra, a partir del texto de José Martínez, que, en este periodo:

Desde el punto de vista de los enfoques teóricos incipientes, que comenzaron o intentaron abrir el campo de los estudios de juventud, podemos señalar -a lo menos- cuatro principales:

- Se analizó a la juventud urbano popular desde el enfoque de la anomia social, a partir de un estudio de campo de Eduardo Valenzuela ${ }^{27}$, y otros que se sumaron, entendiéndola como ciertos niveles de desintegración social, o la disociación entre normas y objetivos culturales (en la perspectiva de Merton), y en la ausencia de normas y de orientaciones de valor (en la perspectiva de Durkheim).

22. Ídem, p. 104.

23. Ídem, p. 105.

24. José Martínez Oyarce, «Discursos sociales y juventud», en Ana Vergara del Solar (edit.), Cartografias de la Juventud, Universidad Diego Portales, Praxis Publicaciones, Santiago de Chile, 2002, p. 11-23.

25. Ídem, p. 14.

26. Oscar DÁVILA, Entrevista vía Internet, noviembre 2004.

27. Eduardo Valenzuela, La Rebelión de los Jóvenes, Sur, Santiago de Chile, 1984. 
- El que comenzó a incluir el enfoque de la exclusión social, como categoría relacional, que daba sus primeros pasos en el análisis sociológico chileno (Javier Martínez ${ }^{28}$ y otros), desde una influencia principalmente francesa.

- El «bienintencionado» enfoque sobre la «juventud en riesgo», que por aquellos tiempos se utilizó como una perspectiva de diagnóstico y fundante de orientaciones de políticas más de tipo promocional y de contestación a las lógicas de acción social de la dictadura; pero ese enfoque se asumió hasta nuestros días, donde las buenas intenciones originales se convirtieron en un enfoque regresivo y controlador, que en buena medida fundamenta la actual política dirigida a jóvenes por el gobierno. Uno de los principales exponentes fue José Weinstein ${ }^{29}$, actualmente ministro de Cultura.

- Un enfoque más de carácter colectivo en la acción social, a diferencia de los anteriores, con un fuerte énfasis en la dimensión agregativa, pudiendo señalarlo como un "enfoque movimientista», intentando aproximarse a la conceptualización de un «movimiento juvenil popular», con una lectura sociopolítica de las prácticas de contestación y resistencia por parte de los jóvenes urbanos populares. Una expresión de aquél lo constituyó la edición de un lindo libro llamado Juventud chilena: razones y subversiones ${ }^{30}$, precisamente a raíz del AIJ, en 1985, editado por tres OSC y que recogió las múltiples expresiones en y desde el quehacer juvenil.

Finalmente, en la década de los noventa, las indagaciones se volcaron en la investigación aplicada y en la evaluación de programas dirigidos a jóvenes, donde la dicotomía social predominante parecía ser la contradicción entre modernización y democratización ${ }^{31}$.

El análisis concluye con la disección de las perspectivas que se están usando en Chile en torno a los estudios de juventud, cruzando dos aproximaciones generales: las orientadas al sujeto (que consideran al fenómeno juvenil a partir de consideraciones individuales y de carácter intrapsicológico) y las orientadas al contexto (que lo estudian a partir de las transformaciones económicas, sociales y culturales propias de la sociedad), con seis perspectivas específicas: demográfica (definición etárea y tratamiento demográfico); ciudadana (jurídica); moratoria (que puede ser de ciudadanía, de identidad, o de exclusión); de modernización (integración o radicalización de la modernidad); crítica (pertenencia a clase social), y generacional (crítica a los modelos adultos) ${ }^{32}$.

$\mathrm{Al}$ respecto, concluye Dávila que sobre todo sigue predominando un enfoque del riesgo, que se conjuga con lo de «juventud peligrosa», en los «vie-

28. Javier MartíneZ y E. VALEnZuela, «Juventud chilena y exclusión social», Revista de la CEPAL, no 29, agosto de 1986.

29. José WeInSTEIN, La juventud urbano poblacional vista desde la sociología, CIDE, Santiago de Chile, 1985.

30. I. AgurTo y otros (ed.), Juventud Chilena: Razones y Subversiones, Eco-Fólico-Sepade, Santiago de Chile, 1985.

31. José Martínez Oyarce, op. cit., p. 17.

32. Ídem, p. 17-22. 
jos/mismos/nuevos» temas de supuestamente lo juvenil, que finalmente son los que copan la opinión pública: "como nosotros decimos, "los cuatro jinetes del Apocalipsis": violencia, delincuencia, drogas y sexualidad ${ }^{33}$.

Por último, el país que más recientemente se ha inclinado a revisar los mapas de lo realizado en materia de juventud es Colombia, con dos estados del arte relevantes. El primero se refiere a la producción de investigaciones realizadas en la ciudad de Bogotá, Colombia ${ }^{34}$, donde sorprende lo reciente de la tradición de los estudios (sólo desde 1994); en este documento, Perea realiza un buen análisis con base a tres aspectos: las elaboraciones en torno a las identidades juveniles (cómo se autodefinen los grupos juveniles), las temáticas abordadas y, finalmente, las tendencias metodológicas y teóricas.

Sobre las identidades, encuentra cuatro formas colectivas: los pandilleros, los buscadores culturales, los comunitarios y los independientes o de identidades múltiples. Cada uno de ellos no exentos de dificultades para delimitar sus fronteras y características; de los primeros, la preocupación sobre sus expresiones violentas, se convirtió en la pregunta ordenadora y obligante; no obstante, las investigaciones realizadas recogen, además de esas prácticas conflictivas, sus posturas frente a la vida cotidiana, la familia y el país, logrando ofuscar la mirada criminalizante para darles voz a sus tragedias y visiones del universo ${ }^{35}$. De los buscadores culturales, los trabajos se centran en dos expresiones musicales, el rap y el rock, expresiones que sirven para generar «comunidades emocionales». En el caso de los comunitarios, los análisis se componen de tres dimensiones: la identitaria, la organizativa y la interrelacional; así desde la estética, la acción solidaria y de su postura frente a la comunidad se posibilita la creación de una sensibilidad conectada al espacio público local ${ }^{36}$. Por otra parte, los textos en torno a los jóvenes de identidades múltiples se refieren a investigaciones que, desde metodologías de historia de vida o encuestas culturales, abordan las vivencias de diversos sectores juveniles.

Por lo que respecta a los estudios centrados en temáticas específicas, destacan los que se refieren a la escuela, la política, los consumos culturales y el trabajo sexual. La revisión de Perea establece que las técnicas más usadas son la historia de vida y el relato testimonial (todo lo contrario de lo que sucede en Uruguay), sólo en tercer término aparece la encuesta, aunque se observa una tendencia a usar técnicas combinadas.

El autor establece como conclusiones la ausencia de «un cuerpo conceptual mínimo compartido y debatido entre los trabajos»; sin embargo, identifica tres desplazamientos en las perspectivas analíticas: «El primero es el que va de la violencia a la identidad; el segundo, el que camina de la política a la cultu-

33. Oscar DÁVILA, entrevista cit.

34. Carlos Mario PereA, «De la identidad al conflicto: los estudios de juventud en Bogotá», en Jesús Martín Barbero y otros (ed.), Cultura y Región, Centro de Estudios SocialesUniversidad Nacional-Ministerio de Cultura, Bogotá, 2000, p. 315-346.

35. Ídem, p. 322-323.

36. Ídem, p. 326-327. 
ra, y, el tercero, el que recorre de las instituciones al sujeto». Lo que significa que se ha ido de un concepto unitario de identidad a un nomadismo que habla del desanclaje de los símbolos; otro que va de la legitimidad otorgada a la voz del sujeto, donde su «verdad» está en la expresión de sus vivencias, y, finalmente, la que va de un sujeto «receptor» a un sujeto autónomo o con la aspiración de serlo ${ }^{37}$.

Un segundo estado de arte en este país, con una cobertura nacional, fue realizado por el Departamento de Investigaciones de la Universidad Central (DIUC), financiado por el Programa Presidencial Colombia Joven, la Agencia de Cooperación Alemana (GTZ) y el Fondo de las Naciones Unidas para la Infancia (UNICEF) de ese país ${ }^{38}$; para lo cual se montó un impresionante dispositivo que implicó estructurar equipos de investigación en seis regiones del país, tomando una base presencial de dieciocho ciudades en las que se concentran los grandes centros de documentación bibliográfica, además de consultas a las principales páginas web relacionadas con el tema de joven y la juventud en ese país. El periodo de indagación implicó lo producido entre de 1985 a 2003, de donde se detectaron 2.457 títulos, elaborándose un resumen analítico especializado (RAE) a 527 documentos seleccionados, que componen la base de datos que se capturó en un software diseñado para tal fin, el SAID (Software de Análisis de Información Documental), de los cuales se hizo un reporte a partir de 507 RAES que se tuvieron a la fecha de corte.

Para este informe se establecieron nueve grandes temáticas (visiones de futuro, familia, cuerpo, educación, inserción sociolaboral, convivencia y conflicto, culturas juveniles versus producción y consumo cultural, participación social y política y políticas públicas), que orientaron la búsqueda de todos los equipos de investigación. Cada temática, a su vez, contempla tópicos con una amplia gama de subtemas relacionados. Adicional a estos temas, se diseñaron dos ejes analíticos transversales: las nociones de sujeto (desglosado en vulnerabilidad y riesgo, peligro social, búsqueda de identidad, cambio social, culturas juveniles y otros) y diversidad (que implicaba clase social, urbano-rural, género, orientación sexual, étnico-racial y discapacidad), a partir de los cuales se indagó sobre la construcción de la juventud desde las investigaciones, para comprender qué imágenes del sujeto joven se estaban produciendo.

De los resultados obtenidos, destacan, en lo cuantitativo: la producción de conocimiento se concentra entre 1993 y 2004 (con un 91,5\% de los textos), alcanzando su nivel más alto entre 1998 y 2000, y, como sucede con México, la mayor parte de la investigación se produce en las zonas urbanas sobre todo en la región Centro, donde se encuentra la capital (48,1\%), y en Antioquia, donde está la ciudad de Medellín, vinculadas a la presencia de la institucionalidad universitaria. Las temáticas más recurrentes son: cuerpo con 19,5\% (estu-

37. Ídem, p. 336-337.

38. Manuel Roberto Escobar C. (coord.), Estado del Arte del Conocimiento Producido sobre Jóvenes en Colombia 1985-2003, Programa Presidencial Colombia Joven, GTZ-UNICEF, Bogotá, 2004. http://www.colombiajoven.gov.co/estado_arte.htm. 
dios relacionados con la corporalidad, la sexualidad y el género), seguida por los de culturas juveniles versus producción cultural $(17,9 \%)$ y de participación social y política $(15,2 \%)$.

Sobre los dos ejes de análisis transversales predominan, en las nociones de sujeto, los conceptos de: vulnerabilidad y riesgo, búsqueda de identidad y cambio social (con un 27,5, un 24,7 y un 17,7\% respectivamente), mientras en el eje de diversidad prevalecen las acotaciones rural/urbano (siendo la mayor parte los estudios que se refieren exclusivamente a jóvenes urbanos), seguido por las divisiones de clase social y de género (con 34,8, 29,5 y 25,8\% para cada uno).

Según el estado del arte colombiano, los estudios de juventud se han concentrado en carreras como psicología, antropología, sociología y ciencias de la educación, sin embargo, recientemente el tema ha empezado a desarrollarse en otras, como ciencias políticas y economía. Una gran proporción de las indagaciones provienen de las tesis de licenciatura, por lo tanto las universidades son el primer productor de conocimiento en esta área, pero las más de las veces sólo lo asumen como un subtema desde las búsquedas disciplinarias y no como líneas de investigación consolidadas. El segundo productor de conocimiento que se identificó fue las organizaciones civiles, que tienen un papel muy importante, sobre todo en las ciudades de Bogotá y Medellín. Sin embargo, el Estado cumple un papel importante en todas las regiones del país, pues convoca y cofinancia ciertos proyectos en el marco de las políticas y acciones que instrumenta, articulándose con entidades universitarias e incluso con procesos de las organizaciones de la sociedad civil (OSC).

El equipo coordinador del documento se sumerge en una serie de discusiones conceptuales muy interesantes, pero que sería largo de exponer en este espacio; sin embargo, algunas de las conclusiones que se adelantan muestran que, a pesar del avance logrado gracias a enfoques novedosos de acercamiento a la compleja realidad juvenil, todavía los estudios en aquel país están inmersos en una fragmentación. La razón, según se expone, es una consecuencia a la diversidad de enfoques que no siempre coinciden entre sí, donde la teoría en ocasiones poco se relaciona con las metodologías adoptadas, lo que produce que acaben siendo meras investigaciones descriptivas. Así mismo, se reproduce en mucho lo que sucede en otros países de la región; por ejemplo, la ausencia de estudios sobre los sectores juveniles medios y altos; la visión lineal y progresiva de las trayectorias de vida juveniles hacia un ideal del sujeto adulto sin una multidimensionalidad; la consideración de la estructura familiar sólo en su papel disfuncional de socialización; las escasas indagaciones novedosas sobre la inserción sociolaboral de los distintos grupos juveniles, etcétera. Termina el estado del arte con una serie de reflexiones y propuestas sobre las políticas necesarias para consolidar el conocimiento sobre juventud basada en tres componentes: la generación de una comunidad académica y la institucionalización de la investigación en el tema; un uso del conocimiento que genera discursos contra los estereotipos y las estigmatizaciones y para la vinculación con los procesos de acción social, y la comunicación del conocimiento producido tanto 
a nivel de circulación y difusión como de estandarización en los centros de documentación para facilitar su consulta.

Como se puede apreciar, estos cuatro estados del arte nacionales y uno local, realizados en países cuya tradición en investigación de juventud ha sido más permanente, nos dibujan un panorama muy paradójico de la investigación latinoamericana en juventud, por una parte, una amplia y diversa producción en distintos campos vinculados a lo juvenil pero, por otra, muy fragmentados y con poca continuidad en los procesos de discusión conceptual y metodológica, aspecto que volveremos a abordar al final del presente documento.

En esta revisión quizá pese la ausencia de mapas parecidos para Argentina y Perú, con una trayectoria similar en este campo o de los países de Centroamérica que, en conjunto, ha desarrollado una producción regional importante, por desgracia no se conocen esfuerzos de este tipo. Por lo tanto, intentaremos dibujar el resto del mapa mediante otros caminos.

\section{Otros lugares de producción de conocimiento}

\section{Las encuestas nacionales}

Las encuestas nacionales de juventud han sido una de las novedades en los últimos veinte años, porque se ha superado la barrera de informar sobre los jóvenes con base a fuentes secundarias (censos, encuesta de hogares, etc.) y se han podido indagar cuestiones más específicas de lo juvenil. Con esto, además, se ha obtenido un mejor panorama que trasciende los universos acotados de las investigaciones particulares y que las más de las veces versan sobre los sectores juveniles más visibles, dejando en la invisibilidad a jóvenes «promedio» o a los desinstitucionalizados (que no estudian ni trabajan), y quizá con un mejor impacto, tanto en las instituciones públicas y privadas como en los medios de difusión masiva.

Con criterios muestrales diversos, con bases teóricas dispersas (algunas francamente inmersas en un total empirismo), con pocas posibilidades de comparación por las temporalidades de aplicación y por el tipo de formulación de los reactivos, hay que reconocer que, a pesar de todo, han empezado a delinear nuevos derroteros en el conocimiento sobre los jóvenes del continente. El camino es largo y sinuoso, porque el verdadero valor de la información estadística es cuando se pueden hacer series de tiempo que muestren el avance o rezago de la población juvenil y esto sólo lo ha logrado Chile (ver cuadro 1).

Un estudio que analizó seis encuestas nacionales de juventud con el fin de encontrar las áreas comparables que ayudaran a elaborar un perfil de los jóvenes en Iberoamérica concluía así su indagación:

La rigurosidad y alto nivel de precisión de las encuestas analizadas son una prueba de la madurez y status que están adquiriendo los ensayos demoscópicos en torno a temas que en el pasado tuvieron una importancia limitada. Por esto, se requiere extender su práctica en los demás países iberoamericanos, conjuntar 
Cuadro1. Encuestas nacionales de juventud realizadas en América Latina, 1998-2003.

\begin{tabular}{llll}
\hline País & Año & Nombre & Muestra \\
\hline Paraguay & 1988 & Encuesta Nacional de la Juventud & 2.200 entre 15 y 24 años \\
Uruguay & 1990 & Encuesta Nacional de Juventud & 6.547 entre 15 a 29 años \\
Chile & 1994 & 1a. Encuesta Nacional de Juventud & 3.792 entre 15 a 29 años \\
Bolivia & 1996 & Encuesta Nacional de Juventudes & 1.774 entre 13 a 24 años \\
Chile & 1997 & 2a. Encuesta Nacional de Juventud & 3.455 entre 15 a 29 años \\
Guatemala & 1999 & $\begin{array}{c}\text { Los jóvenes guatemaltecos } \\
\text { a finales del siglo XXI }\end{array}$ & 2.003 entre 15 y 25 años \\
México & 2000 & Encuesta Nacional de Juventud & $\begin{array}{c}\text { 54.500 hogares con } \\
\text { jóvenes entre 12 a 29 años }\end{array}$ \\
Colombia & 2000 & Encuesta Nacional de Jóvenes & 1.020 entre 12 y 26 años \\
Chile & 2000 & 3a. Encuesta Nacional de Juventud & 3.701 entre 15 y 29 años \\
Chile & 2003 & 4a. Encuesta Nacional de Juventud & 7.189 entre 15 y 29 años \\
\hline
\end{tabular}

esfuerzos para estandarizarlas, al tiempo de desarrollar apartados con diseños compatibles que permitan el seguimiento de distintos aspectos, de tal forma que el conocimiento acumulado no sea desperdiciado. Habría que tomar como ejemplo de contraste los avances desarrollados en el mercado y las empresas, pues han identificando rápidamente los movimientos y cambios en los gustos y preferencias de los jóvenes. Más allá de las realidades y necesidades particulares de cada país, no parece haber mayor problema para acordar el diseño de una encuesta modelo que pueda aplicarse en cualquier país; en todo caso, los problemas pudieran estar en el campo de lo político, donde los intereses y proyectos de las administraciones de los organismos de juventud pueden pesar más que el menor costo beneficio de disponer de una herramienta estándar ${ }^{39}$.

Sabemos que, en 2005, tres países al menos preparan la aplicación de nuevas encuestas nacionales (México, Costa Rica y Nicaragua), quizá sería el momento de establecer ciertos acuerdos, si no para llegar a un diseño modelo como lo propone Navarro, sí para establecer algún número de reactivos comunes que permitan esta comparabilidad tan deseada.

\section{Las publicaciones periódicas}

Otro indicador para medir el avance de la investigación sobre juventud puede ser la generación de revistas especializadas que permitan concentrar los debates y avances de indagación que se vayan realizando; porque sin menoscabo

39. Javier NAVARRO, Las encuestas de jóvenes en Iberoamérica. Un recuento de experiencias recientes, México, 2003 (inédito), p. 55. 
de lo que otro tipo de revistas académicas sobre temas sociales generales puedan publicar, en ocasiones hasta en números monográficos ${ }^{40}$, siempre dificulta esa dispersión en los interesados en el tema que tienen (tenemos) que andar a la caza de lo que se publica en torno a los jóvenes.

Se tienen detectadas seis revistas especializadas en Latinoamérica, tres de ellas han dejado de circular:

- Participación. Revista Uruguaya de Estudios sobre la Juventud, su primer número se publicó en mayo de 1984 en Montevideo, Uruguay, y el último en mayo de 1990. Se produjeron diez números. La editaba el Foro Juvenil de ese país.

- Encuentro. Boletín Latinoamericano de Informaciones sobre Juventud. Su primer número correspondió a julio-agosto de 1986 y el último, a enero-febrero de 1989. Lo editaba el Centro Latinoamericano sobre Juventud (CELAJU), cuya sede estaba también en Montevideo, Uruguay. Se publicaron 16 números.

- Mayo. Revista de Estudios de Juventud, que sólo logró publicar un número, correspondiente a noviembre de 2000. Su editor era la Dirección Nacional de Juventud de Argentina.

Las otras tres revistas que sobreviven son:

- Revista Última Década, que edita desde 1993 el Centro de Investigaciones y Difusión Poblacional de Achupallas (CIDPA), ubicado en Valparaíso, Chile y que a la fecha ha publicado 21 estupendos números (el más reciente correspondiente a diciembre de 2004), además de tener una serie de libros (diez publicados) con diversas temáticas vinculadas a lo juvenil ${ }^{41}$.

- Jovenes. Revista de Estudios sobre Juventud, editada desde 1996 por el Centro de Investigación y Estudios sobre Juventud del actualmente Instituto Mexicano de la Juventud, que va en su número 21 (correspondiente a juliodiciembre de 2004) y que también produce una colección de libros con diecisiete publicaciones realizadas ${ }^{42}$.

40. Por ejemplo, la revista Nueva Sociedad, editada en Caracas, Venezuela, ha dedicado cuando menos dos de sus números al tema juvenil: el número 76, correspondiente al bimestre marzo-abril de 1985, y el número 117, correspondiente a enero-febrero de 1992. De igual forma, la revista colombiana Nómadas dedicó su número 13, de octubre de 2000 al asunto de «la singularidad de lo juvenil». En España se publica poco sobre jóvenes en AL, por lo que destacan dos números de la Revista de Estudios De Juventud, del Instituto de la Juventud de ese país: el número 20 (enero de 1986) y el número 35 (septiembre de 1989).

41. Todos los textos se pueden consultar en sus páginas: www.cidpa.cl y www.cidpa.org

42. Los títulos e índices de estas publicaciones se pueden consultar en www.imjuventud.gob.mx, pronto estarán los documentos íntegros en línea. Además, habría que decir que Jovenes es sucesora de otra publicación que, entre 1981 y 1988, editó la institución gubernamental que en ese momento se llamaba Consejo Nacional de Recursos para la Atención de la Juventud (CREA) y que llevaba el nombre náhuatl impronunciable de: In Telpochtli, In Ichpuchtli. Revista de Estudios sobre Juventud (que significa 'el joven, la joven'). 
- Revista Latinoamericana de Ciencias Sociales, Niñez y Juventud, que desde enero-junio de 2003 vio la luz, aunque, como su nombre lo indica, combina otros temas junto con el de los jóvenes. La editan el Centro de Investigaciones y Estudios Avanzados en Niñez, Juventud, Educación y Desarrollo, el Doctorado en Ciencias Sociales, Niñez y Juventud y el Centro Internacional de Educación y Desarrollo Humano de la Universidad de Manizales, Colombia; a la fecha, ha publicado cuatro números y el más reciente corresponde a julio-diciembre de 2004.

Sólo con revisar y analizar los trabajos que han sido publicados en estos espacios editoriales bastaría para dibujar un complemento del mapa, más detallado de lo que se ha realizado y se realiza en nuestro continente en materia de juventud, pero por desgracia eso rebasa al actual texto.

\section{Grupos de trabajo}

Finalmente, algo habría que decir de los grupos de trabajo o de los espacios que existen en torno a la investigación en jóvenes. Adoptan diferentes nomenclaturas y desarrollan diversas actividades; en este sentido, podemos ubicar a Observatorios de Juventud que existen en Santiago de Chile, en Bogotá, Colombia y el de reciente creación de San José, Costa Rica. Hay otros que funcionan como organizaciones de la sociedad civil, el mismo CIDPA de Chile, que mencionábamos arriba, la resurrección del CELAJU de Montevideo, Uruguay, también ya mencionado; Corporación Región de Medellín, Colombia, ciudad que por cierto ha desarrollado innumerables asociaciones de este tipo y con buena producción investigativa (el Instituto Popular de Capacitación, Paisa Joven, entre otras); la Sociedad Peruana de Adolescencia y Juventud (SPAJ) de Lima, Perú; la Fundación Desafíos de Managua, Nicaragua; en fin, tantos que únicamente podemos enunciar algunos.

En otras ocasiones los grupos se integran mediante redes como la Red Mexicana de Investigadores sobre Juventud; las redes de Sao Paulo y de Río de Janeiro en Brasil (aunque sabemos que en ese extensísimo territorio existen otras), que, independientemente de su adscripción a algún centro académico o universitario tienen puntos de contacto más o menos permanentes. En otros casos, hay instituciones como la Facultad Latinoamericana de Ciencias Sociales (FLACSO), sede Argentina, que tiene un trabajo sistemático y permanente de investigación y formación sobre el tema; o el Centro Interamericano de Investigación y Documentación sobre Formación Profesional (CINTERFOR) de la Organización Internacional del Trabajo (OIT), que se ha especializado en sistematizar un acervo importante en cuestiones de empleo y capacitación juvenil.

Hasta aquí estos trazos, que quizá se conviertan en trozos de una realidad compleja y paradójica, pareciera que la producción investigativa ha sido muy extensa cuando se mira hacia atrás, pero es insignificante cuando se ve el horizonte. 


\section{Un balance provisional}

Una vez delineado este recuento-síntesis, quedarían por apuntar algunas discusiones pendientes y lagunas que todavía quedan por desarrollar, basándome en las mismas conclusiones de los estados del arte reseñados y que pueden aplicarse al estatus que guarda la investigación sobre juventud en el continente.

Insisto, como ya lo hice en algún otro momento ${ }^{43}$, que todavía no es posible hablar propiamente de un campo de conocimiento sobre juventud en AL, ya que, por lo general, entre los diferentes agentes vinculados a él no se construyen los mecanismos necesarios para desarrollarlo ${ }^{44}$. Así, tenemos que, por parte de los investigadores, en la mayoría de los casos no hay una recuperación de lo que otros escriben (para discutir o asumir) ${ }^{45}$; por parte de las instituciones universitarias y/o académicas, sigue siendo secundario el tema y, por lo tanto, no hay o son muy escasos los apoyos permanentes de impulso a la temática (investigación y difusión); en cuanto a las instancias públicas (gubernamentales o de la sociedad civil), tampoco existe una tradición de acercamiento e intercambio entre los que producen conocimiento y quienes toman decisiones, que, en el mejor de los casos, los hace caminar en paralelo y, en el peor, en sentidos opuestos.

Da la impresión que en nuestro continente los que producen conocimiento sobre los jóvenes es gente muy aislada. En los estados del arte revisados, se acentúa la necesidad de generar un proceso colectivo que permita una construcción regional y compartida de explicaciones e interpretaciones mejor articuladas, como lo afirma Perea, cuando menos para que exista «un corpus conceptual mínimo» ${ }^{46}$. El avance que han significado las encuestas nacionales de juventud, en cuanto fuentes empíricas, tiene como Jano, su otra cara, el endiosamiento del dato por el dato mismo, que no supera el nivel de la enunciación sino parte de una teoría que la contextualice.

Como consecuencia de lo anterior, tenemos, por un lado, todavía una preponderancia de los enfoques empiristas, donde la escasa explicitación y discusión conceptual de muchos estudios queda sólo a nivel de delimitación etaria (que, por cierto, tampoco a este nivel se ha llegado a un acuerdo $)^{47}$; pero, por

43. Algo ya habíamos planteado en Carme Gómez-Granell y otros, «Introducción. De redes y retos», en Rossana Reguillo y otros, Tiempo de Hibridos, op. cit., p. 11-15.

44. Apropiándonos el concepto de Bourdieu en torno al campo social, éste se construye relacionalmente y de acuerdo con un capital específico que detentan los agentes que juegan en ese espacio social, que se define y se negocia históricamente. Andrés GarCía IndA, «Introducción», en Pierre Bourdieu, Poder, derecho y clases Sociales, Desclée de Brouwer, Bilbao, 2001, p. 14-15.

45. A Braslavsky le llama la atención la poca presencia de polémicas y discusiones en la etapa más reciente que ella analiza, op. cit., p. 40 .

46. Carlos Mario PereA, op. cit., p. 341.

47. Esto sucede no sólo en el comparativo entre países de la región; sino en el interior del mismo país, las fuentes estadísticas varían en los estratos etáreos cuando se trata de salud, de educación o de trabajo, por lo tanto, no se pueden articular. 
otro lado, los «recién llegados» al campo (de nuevo sigo a Bourdieu) tienen que partir de cero, dado que no existe una tradición que los ubique en el mapa conceptual que se ha ido construyendo a lo largo del tiempo.

Si bien se puede observar un avance desde el punto de vista teórico sobre los jóvenes en lo que Lovesio y Viscardi llaman el paso de ser «un segmento social» a una categoría sociológica con elementos que estructuran socialmente sus relaciones ${ }^{48}$, esto no se ha dado en todas las temáticas; por ejemplo, en el caso de los temas vinculados a la salud, aunque con honrosas excepciones, se privilegian los enfoques epidemiológicos; en los de empleo o educación, los estructural-funcionalistas (como el de moratoria social), y, en el otro extremo, los enfocados en estudios culturales, en ocasiones desaparecen la estructura.

El predominio de lo disciplinario en las investigaciones de juventud y el enfoque normativo (doble disciplinario) que todavía no se les ha despojado a muchas de ellas, son un reto para los siguientes años; hay que «impensar las ciencias sociales», nos dice Wallerstein, «debido a que muchas de sus suposiciones —engañosas y constrictivas, desde mi punto de vista — están demasiado arraigadas en nuestra mentalidad ${ }^{49}$, y esto es particularmente fundamental para consolidar el campo de investigación de lo juvenil.

Pero esto también implica que los demás agentes del campo se involucren, es decir, las instituciones académicas deben constituir espacios más estables y con apoyos de formación y difusión, así como las gubernamentales abrir sus programas al diálogo con la producción de conocimiento, dado que, en función de la toma de decisiones: "no hay mejor idea, que una buena teoría». Sobre todo la responsabilidad que las instituciones internacionales-regionales tienen de fomentar espacios de discusión colectiva para fortalecer una construcción identitaria de los interesados en la investigación de juventud ${ }^{50}$, y el establecimiento de ciertos acuerdos base para un desarrollo más articulado. Para que, finalmente, como propone el grupo de Escobar ${ }^{51}$ :

Se plantean políticas de juventud que vayan más allá de la emergencia social, que también debe ser atendida, y apunten a lo general de las condiciones de producción de lo juvenil en nuestra propia sociedad. Una política de los capitales sociales que considere la dimensión política de los-as jóvenes más allá de su incorporación en los procesos de participación institucional y les provea de capacidades de reformulación y transformación de lo social. Que también contemple los capitales simbólicos en tanto constructores de imaginarios y representaciones sobre el joven; capitales culturales que no sólo exoticen las estéticas y sensibilidaes juveniles sino que contemplen su lugar dentro de la

48. Op. cit., p. 104-105.

49. Immanuel Wallerstein, Impensar las Ciencias Sociales, Siglo XXI-UNAM, México, 1998, p. 3.

50. Como lo tenía la OIJ, que, en sus primeros años, bianualmente se reunían funcionarios, investigadores y asociaciones juveniles a discutir y dialogar temas específicos.

51. Manuel Roberto EsCOBAR C. (coord.), op. cit., p. 228. 
construcción de lo social; y unos capitales económicos que posibiliten acceso a amplios mundos y cosmovisiones.

Ahora podemos responder a la pregunta que nos hacíamos al inicio de este texto. En AL todavía no podremos generar un pensamiento latinoamericano sobre juventud, mientras no construyamos este campo del conocimiento, involucrando a todos los agentes, para lograr espacios dialógicos y de interactividad que consoliden el capital que existe de manera fragmentaria. Un inicio pueden ser estas cartografías o mapas de las actuaciones y representaciones que la investigación hace para captar, no para fijar, sino más bien «instrumento de navegación que es la navegación misma [...] Mapa que no es una copia del fenómeno, sino el registro, el texto de su funcionamiento en tanto práctica de su propio movimiento" 52 .

En este sentido, para finalizar, habría que estar atentos a los procesos transformados e instalados en el campo de lo juvenil, que marcarán las tendencias en los próximos años en el continente para encararlos adecuadamente. Algunas de estas tendencias serán:

- La dislocación de las ciudadanías juveniles, generadas por los flujos migratorios principalmente, pero también por los nuevos sistemas de comunicación electrónica, que generan procesos de hibridación y de desterritorialización y reterritorialización muy complejos.

- La desubicación de los procesos escolares formales en la vida de los jóvenes, quienes poseen en ocasiones un mejor manejo cultural de los avances tecnológicos y de comunicación que sus profesores; pero que va más allá, dado que la vida no pasa por la escuela, ni la escuela por la vida de las nuevas generaciones.

- La posibilidad de una transformación en la concepción y la práctica del trabajo, ante la acentuación de los procesos de exclusión laboral y de explotación de la mano de obra juvenil, para convertirse en un proceso de resistencia a la lógica capitalista de trabajo = dinero, que cada vez es menos significativa para los jóvenes (pensar, por ejemplo, el mercado de trabajo no estructurado o informal, o el narcotráfico) y viceversa, que se ejercía por el disciplinamiento de tiempo y lugar.

- El nomadismo de las relaciones juveniles de género (que puede ir de lo tradicional a lo innovador, pero también a la inversa), en condiciones que marcan a las mujeres jóvenes latinoamericanas con mejores promedios educativos que sus pares varones; con perspectivas más acertivas sobre su vida; con mayores posibilidades de ocuparse en un empleo, etc.

- Un retorno y/o reforzamiento, por parte de las nuevas generaciones, al pensamiento mágico-religioso, ante la incertidumbre que los envuelve, y que

52. Mauricio Sepúlveda G., «Reflexiones en torno a la distinción entre 'estudios sobre y desde los jóvenes’: hacia una cartografía deseante», en Ana Vergara del SOlarp. 50. 
fácilmente es presa de fundamentalismos y localismos ante los «otros» diferentes.

- Los símbolos y prácticas que está generando la nueva socialidad juvenil o esos nuevos modos-de estar-juntos, donde el cuerpo, las sustancias, los tiempos, los espacios, son resignificados y que la investigación debe acompañar, sin disciplinar.

\section{Bibliografía}

Nota: además de la bibliografía utilizada para la elaboración de este trabajo, se enumera una muestra de algunas publicaciones que pueden ayudar a conocer mejor la producción en América Latina.

ABaUnZA, Humberto; ANDino, Ricardo (2003). Juventud y nuevos desafios en Nicaragua. Managua: Fundación Desafíos.

Abramo, Helena Wendel y otros (orgs.) (2002). Juventude em Debate. Sao Paulo: Cortez Editora.

Agurto, I. y otros (ed.) (1985). Juventud Chilena: Razones y Subversiones. Santiago de Chile: Eco-Fólico-Sepade.

ANGUlo, H. Mario (1995). La Juventud Venezolana. Informe general, versión resumida, Caracas: Ministerio de la Familia. Hacia el Desarrollo Social.

BRASLAVSKY, Cecilia (1989). "Estudios e investigaciones sobre juventud en América Latina: balance y perspectivas». En RODRÍGUEZ, Ernesto; OTTONE, Ernesto (comp.). Mitos, certezas y esperanzas: Tendencias de las investigaciones sobre juventud en América Latina. Montevideo: CELAJU-UNESCO, p. 17-48.

Bourdieu, Pierre (2001). Poder, derecho y clases sociales. Bilbao: Desclée de Brouwer. Caccia-Bava, Augusto; FeiXa PÁmpols, Carles; Gonzáles Cangas, Yanko (2004). Jovens na América Latina. Sao Paulo: Escrituras/CEBRIJ.

Camacho, Daniel; Menjílar, Rafael (coords.) (1985). Movimientos populares en Centroamérica. San José, Costa Rica: Biblioteca Perspectivas de América Latina. UNU/FALCSO/ISSUMAN. Colección Seis.

CÁNEPA, María Angela (1993). Esquinas, rincones, pasadizo. Bosquejos sobre juventud peruana. Lima: Instituto Bartolomé de las Casas. Rimac, CEP.

CÁNEPA, María Angela y otros (1993). Los nuevos hijos del sol. Cusco: Allpanchis, Instituto de Pastoral Andina.

Carvalho, Alysson; Salle, Fátima; Guimarāes, Marilia (orgs.) (2003). Adolescência. Belo Horizonte: UFMG.

CASTElo BRAnCO, Maria Teresa (2003). Jovens sem-terra. Identidades em movimento. Brasil: UFPR.

CASTRO, Carmen (ed.) (2001). Jóvenes: ¿En busca de una identidad perdida? Santiago de Chile: Universidad Católica Cardenal Raúl Silva Henríquez, Vicerrectoría Académica, Dirección de Investigación y Extensión DIE, Centro de Estudios en Juventud (CEJU). Serie Jóvenes núm. 1.

CEPAL (1990). Problemas y perspectivas de la integración de los jóvenes en las sociedades latinoamericanas. Toledo, mayo de 1990. 
Chávez de PAz, Dennis (1989). Juventud y Terrorismo. Características sociales de los condenados por terrorismo y otros delitos. Lima: Instituto de Estudios Peruanos.

CINTERFOR-OIT (1988). Empleo y capacitación de jóvenes en América Latina: situación, perspectivas y politicas alternativas. Montevideo.

- (1998). Juventud, educación y empleo. Montevideo.

CORPORACIÓN REGIÓN (1993). Ser joven en Medellín. Medellín.

Corrales, Jeaneth; AbaunZa, Humberto; Andino, Ricardo (2003). Mujeres jóvenes en escena. Managua: Desafíos Fundación.

CORTÁZAR, Velarde Juan Carlos y otros (2001). Políticas de Juventudes: por la igualdad de oportunidades. Perú: Sociedad Peruana de Adolescencia y Juventud (SPAJ).

CruZ, Neto Octavio; RaSGa Moreira, Marcelo; Mazzei SuCENA, Luis Fernán (2001). Nem Soldados Nem Inocentes, Juventude e tráfico de drogas no Rio de Janeiro. Rio de Janeiro: Editorial Fiocruz.

Cubides C., Humberto J. y otros (eds.) (1998). Viviendo a toda. Jóvenes, territorios culturales y nuevas sensibilidades. Santafé de Bogotá: Siglo del Hombre Editores. Fundación Universidad Central.

DÁvalos, Guillermo; CAJíAs, Huáscar (1995). «Juventud boliviana: utopías y realidades». El Debate Boliviano, no 12. La Paz: Seamos, p. 53-92.

DÁvila, Oscar (2004). Entrevista vía Internet. Noviembre de 2004.

De LEÓn, Eduardo; LASIDA, Javier; ROdRÍGUEZ, Ernesto (coord.); RUÉTALO, Jorge (1987). Juventud, empleo y capacitación profesional en el Uruguay: balance y perspectivas. Montevideo.

DONAS, Burak Solum (comp.) (2001). Adolescencia y Juventud en América Latina. Cartago, Costa Rica: Libro Universitario Regional LUR.

Escobar C., Manuel Roberto (coord.) (2004). Estado del arte del conocimiento producido sobre jóvenes en Colombia 1985-2003. Bogotá: Programa Presidencial Colombia Joven. GTZ-UNICEF.http://www.colombiajoven.gov.co/estado_ arte.htm.

FeIXA, Carles (1998). El Reloj de Arena: Culturas juveniles en México. México: Causa Joven. CIEJ.

GingOld, Laura; SÁnCHEZ, Marcelo P.; AuYero, Javier; DelPinO, Nena, WorTMAn, Ana; GonZÁleZ, Enrique; SAlCEdo, Adriana; Umbría ACOSTA, Luis (1992). Juventud, hábitos y fluctuaciones. Caracas: VII Certamen Latinoamericano de Ensayo Político, Nueva Sociedad, no 117, enero-febrero de 1992.

GonÇAlVES DE Asis, Simone; CONSTANTINO, Patricia (2001). Filhas do Mundo, infração juvenil feminina no Rio de Janeiro. Río de Janeiro: Editorial Fiocruz.

GURRIERI, A. y otros (1971). Estudios sobre la juventud marginal latinoamericana. México: Siglo XXI.

Hermo, Javier; BALARDini, Sergio; CASTILlo, José (1994). Primer Informe de Juventud de la Ciudad de Buenos Aires. Buenos Aires: FLACSO. Serie de Documentos e Informes de Investigación, no 173.

LOVESIO, Beatriz; VISCARDI, Nilia (2003). «Los estudios de la mujer y de los jóvenes en la construcción del conocimiento sociológico uruguayo». Revista de Ciencias Sociales, año XVI, no 21. Montevideo: Universidad de la República. Facultad de Ciencias Sociales. Departamento de Sociología, agosto de 2003, p. 79-109.

MARGULIS, Mario (ed.) (1995). La juventud es más que una palabra. Ensayos sobre cultura y juventud. Buenos Aires: Biblos.

Marín, Martha; MuÑoz, Germán (2002). Secretos de mutantes. Música y creación en las culturas juveniles. Colombia: Siglo del Hombre Editores. 
MARTín-BARBERO, Jesús y otros (2000). Umbrales: Cambios culturales, desafios nacionales y juventud. Medellín: Corporación Región.

MarTínez Oyarce, José (2002). «Discursos sociales y juventud». En VerGara DeL Solar, Ana (ed.). Cartografías de la Juventud. Santiago de Chile: Universidad Diego Portales. Praxis Publicaciones, p. 11-23.

MartíneZ, Javier; VAlEnZuela, E. (1986). «Juventud chilena y exclusión social». Revista de la CEPAL, no 29, agosto de 1986.

Medina, Andrés; VAldés, Alicia (comp.) (1995). Ni adaptados, ni desadaptados... sólo jóvenes. Siete propuestas de desarrollo juvenil. Santiago de Chile: PIIE.

MEDINA CARRASCO, Gabriel (comp.) (2000). Aproximaciones a la diversidad juvenil. México: El Colegio de México.

Mejía, Velásquez; Londoño Rendón, Carlos Enrique; Granda Marín, Alberto (1994). La Juventud de Medellín y la Construcción de la Democracia. Medellín: Universidad Pontificia Bolivariana. Departamento de Formación Humanista.

MéndeZ-Carrión, Amparo; Bustamante, Fernando (1995). "Purposes and methods of Intraregional Comparison». En SMITH, Meter H. (coord.). Latin America in Comparative Perspective: New approaches to methods and analysis. Westview Press, Inc., p. 59-80.

NAVARRO, Javier (2003). Las encuestas de jóvenes en Iberoamérica: Un recuento de experiencias recientes, México (inédito).

OIJ-CEPAL (2004). La juventud en Iberoamérica: Tendencias y urgencias. Santiago de Chile.

OIJ-CIID (1994). Proyecto de investigación: Politicas de Juventud en América Latina: evaluación y reformulación. Montevideo (inédito).

ONU-CEPAL (2000). Juventud, población y desarrollo en América Latina y el Caribe: Problemas, oportunidades y desafios. Santiago de Chile: CELADE, CEPAL, FNUAP.

Perea, Carlos Mario (2000). «De la identidad al conflicto: los estudios de juventud en Bogotá». En MarTín Barbero, Jesús y otros (eds.). Cultura y región. Bogotá: Centro de Estudios Sociales. Universidad Nacional. Ministerio de Cultura, p. 315-346.

PÉREZ IsLAS, J.A. (coord.) (2000). Jóvenes: Una evaluación del conocimiento: La investigación sobre juventud en México 1986-1999 (2 tomos). México: IMJ-CIEJ (edición corregida y aumentada).

Pérez Islas, J.A.; MALdONADO Oropeza, Elsa P. (coords.) (1996). Jóvenes: Una evaluación del conocimiento. La investigación sobre juventud en México 1986-1996 (2 tomos). México: Causa Joven. CIEJ.

Pérez Islas, J.A.; Urteaga Castro-Pozo, Maritza (coords.) (2004). Historias de los jóvenes en México: Su presencia en el siglo XX. México: IMJ-AGN.

PÉrEZ IsLAS, J.A.; ZERMEÑo, Sergio (1989). «La juventud mexicana: una discusión sobre su conocimiento». En RodríGUEZ, Ernesto; OTTONE, Ernesto (comp.). Mitos, certezas y esperanzas: Tendencias de las investigaciones sobre juventud en America Latina. Montevideo: CELAJU-UNESCO, p. 119-145.

PONTES, Fraga Paulo Cesar; Silva IUliOANELli, Jorge Atilio (orgs.) (2003). Jovens em Tempo real. Río de Janeiro: DP\&A Editora.

Rama, Germán W.; FilgueIra, Carlos (1991). Los jóvenes de Uruguay. Esos desconocidos. Montevideo: Análisis de la Encuesta Nacional de Juventud de la Dirección General de Estadística y Censos, Comisión Económica para América Latina y el Caribe, oficina de Montevideo (CEPAL), diciembre de 1991. 
Reguillo, Rossana (1997). «Culturas Juveniles. Producir la identidad: un mapa de interacciones». En JOVENes: Revista de Estudios sobre Juventud. México DF: Causa Joven-CIEJ. Cuarta época, año 2, no 5 . julio-diciembre, 1997, p. 12-31.

- (2000). Emergencia de culturas juveniles: Estrategias del desencanto. Buenos Aires: Grupo Editorial Norma, Enciclopedia Latinoamericana de Sociocultura y Comunicación.

Reguillo, Rossana y otros (coords.) (2004). Tiempo de hibridos. Entresiglos, Jóvenes México-Cataluña. México: SEP-IMJ-SGJ-CIIMU.

Rodríguez Carrano, Paulo César (2002). Os jovens e a cidade: Identidades e prácticas culturais em Angra de tantos reis e rainhas. Río de Janeiro: Reulume Dumara/FAPERJ.

- (2003). Juventudes e cidades educadoras. Sao Paulo: Editora Vozes.

RodrígUEZ, Ernesto; DABEZIES, Bernardo (1991). Primer Informe sobre la Juventud de América Latina, 1990. Madrid: Conferencia Iberoamericana de Juventud.

RODRÍGUEZ, Ernesto y otros (1998). La juventud latinoamericana en los procesos de globalizacón: Opción por los jóvenes. En HÜNERMANN, Peter; ECKHOLT, Margit (eds.). Buenos Aires: Intercambio Cultural Alemán-Latinoamericano (ICALA)/FLACSO/EUDEBA.

Rospiglioso, Fernando (1987). Los jóvenes obreros de los 80: inseguridad, eventualidad y radicalismo. Documento de trabajo núm. 18. Lima: Instituto de Estudios Peruanos. Serie Sociología/Política no 3.

- (1988). Juventud obrera y partidos de izquierda: De la dictadura a la democracia. Lima: Instituto de Estudios Peruanos. Serie Ideología y Política no 4.

SEPÚlVEDA G., Mauricio (2002). «Reflexiones en torno a la distinción entre "estudios sobre y desde los jóvenes": hacia una cartografía deseante». En VERGARA DEL SOLAR, Ana (ed.). Cartografías de la Juventud. Santiago de Chile: Universidad Diego Portales, Praxis Publicaciones, p. 41-57.

SePúlvedA G., Mauricio y otros (comps.) (2005). Nuevas geografías juveniles: Transformaciones socioculturales. Santiago de Chile: Universidad Diego PortalesINJUV.

STERN, Claudio; GARCÍA, Elizabeth (2000). Acervo documental sobre sexualidad y salud reproductiva de adolescentes y jóvenes. Documentos de Trabajo no 2. México: Colegio de México. Programa Salud Reproductiva y Sociedad.

TORRACA DE BRITO, Leila Maria (coord.) (2000). Jovens em conflito com a lei. Río de Janeiro: UERJ.

TORRES-RIVAS, Edelberto y otros (1989). Escépticos, narcisos, rebeldes: 6 estudios sobre la juventud. San José: FLACSO/CEPAL.

Urán A. Omar Alonso (1995). La ciudad de los jóvenes. Una mirada desde Medellín. Medellín: RE Lecturas/Instituto Popular de Capacitación.

- (2000). La ciudad en movimiento: Movimientos sociales, democracia y cultura en Medellín y el área metropolitana del valle de Aburrá. Medellín: Instituto Popular de Capacitación, mayo de 2000.

ValenZuela, Eduardo (1984). La rebelión de los jóvenes. Santiago de Chile: Sur.

VALENZUELA, José Manuel. "Culturas juveniles. Identidades transitorias» (1997). JOVENes. Revista de Estudios sobre Juventud. México, DF: Causa Joven-CIEJ, cuarta época, año $1, \mathrm{n}^{\circ} 3$, enero-marzo, p. 12-35.

- (1997). Vida de barro duro: Cultura popular y graffiti. Guadalajara: Universidad de Guadalajara. Colegio de la Frontera Norte.

VAlenZUela, José Manuel; GonZÁLEZ, Gloria (1999). Oye Como va: Recuento del rock tijuanense. México: Instituto Mexicano de la Juventud-CIEJ. 
VOLPI, Mario (2001). Sem Libertade, Sem Direitos: A privação de liberdade na percepção do adolescente. Sao Paulo: Cortez Editora.

WAISELFISZ, Julio Jacobo (coord.) (2003). Relatório de Desenvolvimento 2003. Brasil: UNESCO.

WALLERSTEIN, Immanuel (1998). Impensar las ciencias sociales. México: Siglo XXI. UNAM.

WeINSTEIN, José (1985). La juventud urbano poblacional vista desde la sociología. Santiago de Chile: CIDE.

José Antonio Pérez Islas. Sociólogo mexicano, con especialización en políticas de juventud por parte de la Organización de las Naciones Unidas. Coordinador general del Centro de Investigación y Estudios sobre Juventud del Instituto Mexicano de la Juventud y director de la revista Jóvenes, Revista de Estudios sobre Juventud. Correos electrónicos: japerez@imju ventud.gob.mx; perezislas@yahoo.com 